\title{
Circulación del conocimiento sobre Canarias a la luz de cuatro tesis doctorales alemanas (1869-1894)
}

\section{Marcos Sarmiento Pérez}

\section{(2) OpenEdition}

1 Journals

Edición electrónica

URL: https://journals.openedition.org/cher/494

DOI: $10.4000 /$ cher.494

ISSN: 2803-5992

Editor

Presses universitaires de Strasbourg

Edición impresa

Fecha de publicación: 10 junio 2021

Paginación: 83-100

ISBN: 9791034400928

ISSN: 1968-035X

Referencia electrónica

Marcos Sarmiento Pérez, «Circulación del conocimiento sobre Canarias a la luz de cuatro tesis doctorales alemanas (1869-1894)», reCHERches [En línea], 26 | 2021, Publicado el 15 septiembre 2021, consultado el 20 noviembre 2021. URL: http://journals.openedition.org/cher/494 ; DOI: https://doi.org/ 10.4000/cher.494 


\title{
Circulación del conocimiento sobre Canarias a la luz de cuatro tesis doctorales alemanas (1869-1894)
}

\author{
Marcos Sarmiento Pérez*
}

$\mathrm{E}^{n}$ el último tercio del siglo $\mathrm{xIX}$ se realizaron cuatro tesis doctorales relacionadas con las islas Canarias en las universidades alemanas de Berlín, Estrasburgo, Halle y Bonn, sobre: embriología, antropología, economía y geología. Considerando el consabido interés que la ciencia alemana mostraba entonces por diversas ramas científicas en Canarias, partimos de la hipótesis de que aquellas tesis reflejan el transvase y la circulación de conocimientos adquiridos previamente en las Islas por naturalistas, científicos, académicos y eruditos alemanes. Consecuentemente, nos proponemos indagar en los contextos que indujeron a cuatro jóvenes a elegir Canarias para iniciarse en la investigación, despejando varias incógnitas: situaciones que propiciaron la elección, profesores que influyeron y guiaron las investigaciones, relaciones de los implicados con Canarias y entre sí, instituciones en las que estudiaron y trabajaron, trayectorias personales de los candidatos, etc. Esto nos permitirá arrojar luz sobre las redes que se fueron tejiendo en relación con Canarias durante un cuarto de siglo (1869-1894).

Teniendo en cuenta que la primera de las tesis se presentó en 1869, apenas dos años antes de la unificación de Alemania (1871), y que un reglamento ministerial del 15 de abril de 1872 impulsó una reforma en el acceso al grado de doctor que eliminara desigualdades entre las universidades alemanas, aunque respetando peculiaridades que no contraviniesen dicho reglamento (Baumgart, 1885: IX), nos fijaremos también en las similitudes y diferencias en las cuatro tesis objeto de estudio. Previamente, nos parece oportuno recordar los orígenes de las universidades en las que se realizaron la tesis. En primer lugar, la Königliche Friedrich-Wilhelms-Universität zu Berlin ${ }^{1}$ (a partir de 1949, Humboldt-Universität zu Berlin) se había fundado en 1810, impregnada de la

\footnotetext{
* Marcos Sarmiento Pérez, Universidad de Las Palmas de Gran Canaria. ORCID: 00000002-0704-0043, <marcos.sarmiento@ulpgc.es>.

1 Por el rey de Prusia Friedrich Wilhelm III (1797-1840).
} 
reforma impulsada por Wilhelm von Humboldt, a la sazón ministro de Cultura prusiano, por la que el profesor universitario había de enseñar lo que sabía e investigar en lo que desconocía (Paletschek 2002: 183-184). Bajo el paraguas del Ministerio del Interior prusiano había quedado también la Martin-LutherUniversität Halle-Wittenberg desde que en 1817 surgiera de la fusión de la Leucorea, fundada en Wittenberg en 1502, y la Friedrichs-Universität, en 1694 (Koch 1839: 528-529). A su vez, marcada por la misma visión humboldtiana, había nacido en 1818 la Königliche Rheinische Friedrich-Wilhelms-Universität Bonn, que pasaría a integrar las seis universidades del Estado prusiano (con Berlín, Breslavia, Greifswald, Halle y Königsberg) (Koch 1839: 1). Finalmente, la Universidad de Estrasburgo -que existía desde 1621- se había refundado con el nombre de Kaiser-Wilhelms-Universität Strassburg en 1872, al finalizar la guerra franco-alemana, y también estaba sometida a la Cancillería imperial en Berlín².

Comoquiera que las tesis aportan poca información sobre los doctorandos y sus directores, nos proponemos consultar bio-bibliografías al respecto, catálogos (Verzeichnisse) de docentes y estudiantes de las universidades en cuestión y bibliografía general en torno a las universidades alemanas ${ }^{3}$. Para la presentación de las cuatro tesis hemos adoptado el criterio de la cronología.

\section{De las tesis y su finalidad}

Antes de indicar las razones para hacer una tesis en el espacio geográficotemporal que nos ocupa, nos parece oportuno dilucidar algunos pormenores más generales. Si bien originalmente se manejó el concepto de disputatio, es decir, el debate científico entre doctos -núcleo del examen conducente al doctorado, en el que se abordaba oral y públicamente una o varias materiasmás tarde se empezó hablar de dissertatio, cuando se anunciaban por escrito las tesis defendidas por el candidato (Rasche 2018: 294; Marti 2018: 272-273). Las disertaciones se imprimían en unas 20-30 páginas en lengua latina, que aún, hasta el tercer cuarto del siglo XIX, en el ámbito germanoparlante se consideraba el medio de comunicación entre eruditos (Marti 2018: 271), y cuyo uso en las disertaciones fue obligatorio, al menos en Prusia, hasta $1867^{4}$. A tenor de ello, todas las tesis recogían, junto al título, la leyenda «Disertación inaugural para la obtención del grado de doctor». En efecto, la disertación inaugural era el texto

2 Recordemos que apenas medio siglo después, el 22 de noviembre de 1919, se tornó en la Université de Strasbourg.

3 Lamentablemente, la situación vivida en los últimos meses por la covid-19, que ha provocado el cierre de bibliotecas y archivos, nos ha impedido consultar, salvo alguna excepción, el intercambio epistolar de los implicados.

4 Un decreto ministerial prusiano de aquel año ya permitió a los estudiantes de Medicina presentar y publicar sus tesis en alemán. En las facultades de Filosofía, el latín siguió siendo obligatorio hasta 1879 , no obstante, aún con la salvedad de que quienes lo hicieran en alemán habrían de acreditar, mediante examen oral, tener suficientes conocimientos de latín (Marti 2018: 280). 
que se presentaba para la ceremonia solemne que daba lugar a la promoción, o sea, al ascenso al grado doctor en una facultad. Stricto sensu, "promoción" significaba que el candidato, que defendía sus tesis desde la silla inferior, subía (promovía) luego a recibir la calificación a la silla superior (cf. Baumgart 1885: 160). Cabe añadir que la obligación de publicar la disertación como «el medio más efectivo para garantizar su calidad» se extendió gradualmente por toda Alemania a partir de 1877 (Rasche 2018: 296).

¿Pero cuál era realmente la finalidad de una tesis? Más allá de que las tasas académicas abonadas por los candidatos representaban una importantísima fuente de ingresos para las universidades, y de que en la próspera sociedad decimonónica el título de doctor daba prestigio y favorecía la carrera profesional (cf. Rasche 2018: 295), existían motivos más evidentes: por un lado, desde finales del siglo XVIII, en las facultades de medicina se hizo obligatoria para combatir la competencia que para los médicos representaban los curanderos y charlatanes (Rasche 2018: 296)5; por otro, en la mayoría de las universidades alemanas, el título de doctor era indispensable para ingresar en la carrera docente -inicialmente con el grado de Privatdozent (cf. Koch 1839: 289; Baumgart 1885: XIII, 161); y no menos importante, la tesis era la entrada solemne a una vida de dedicación a la ciencia y el medio para llegar a la publicación científica. Horn (1893: 118) lo explicaba señalando que «quien asimilaba la ciencia era un docto y quien la ejercía en alguna posición profesional era un doctor».

\section{La tesis de Hermann Fol. Embriología}

Cronológicamente, la primera de las tesis que nos ocupan es la de Hermann Fol (1845-1892), presentada y defendida en la Facultad de Medicina de la Universidad de Berlín el 17 de agosto de 1869, sobre la anatomía e historia evolutiva de cuatro especies de ctenóforos $^{6}$, con una de las cuales (Vexillum parallelum Fol) inmortalizó su nombre en la ciencia. La génesis de aquella tesis, una de las pioneras en presentarse en alemán, y no en latín, como había sido habitual hasta apenas dos años antes, fue la siguiente: Fol, que había nacido en Saint-Mandé, cerca de París, aunque sus padres eran de Ginebra, ciudad donde luego cursaría el bachillerato y sus dos primeros años en Zoología, había ido a Jena atraído por el anatomista-embriólogo Carl Gegenbaur y por Ernst Haeckel, flamante exponente del darwinismo en el continente europeo tras la aparición, en 1859, de la revolucionaria teoría darwiniana. En la universidad sajona estudió cuatro semestres, hasta el otoño de 1866, cuando, invitado por Haeckel, lo

5 En este contexto cabe destacar también el decreto promulgado por Napoleón en 1803 para las universidades francesas, según el cual, el ejercicio de la medicina se supeditaba a la obtención de un doctorado (Chesa Ponce 2015: 120). De hecho, los cerca de 40 canarios que estudiaron Medicina en Francia en el siglo XIX regresaron provistos de una tesis doctoral (cf. Chesa Ponce 2015).

6 Celentéreos marinos que, por su vida planctónica, recuerdan a ciertas medusas. 
acompañó a la isla de Lanzarote para investigar animales marinos inferiores en el contexto del evolucionismo. En las tranquilas aguas de la bahía de Arrecife, el joven suizo capturaba ejemplares de ctenóforos, cuyo desarrollo analizaba en el rudimentario laboratorio que Haeckel y sus acompañantes ${ }^{7}$ habían instalado, sin imaginar que aquellas incipientes investigaciones darían lugar tres años después al texto de su disertación.

De vuelta a Europa, cursó un semestre en la Universidad de Heidelberg, otro en Zúrich y el último en Berlín (Fol 1869: 13). Terminados los estudios en 1869, tenía necesariamente que obtener el grado de doctor para ejercer la medicina. A diferencia de los otros tres doctorandos, Fol no indica quién lo impulsó a realizar su trabajo, que dedica a su venerado Gegenbaur. Pero es evidente que el silencio clama por Haeckel, que había guiado sus investigaciones en Lanzarote -intuimos que debido a la enturbiada relación en la Isla, donde el sabio de Jena se vio obligado a reprenderlo en varias ocasiones por su insolente comportamiento (cf. Sarmiento Pérez 2011: 50). Sea como fuere, no parece que el caso de Fol fuera inusual, pues también Gustav Schwalbe (1844-1916), que igualmente se había doctorado en Medicina en Berlín en 1866, y que luego dirigió la tesis de Franz Keibel en Estrasburgo, eligió por su cuenta el tema de la suya (Keibel 1916: 210).

En cualquier caso, es evidente que Fol aprovechó para su «InauguralDissertation» las observaciones y los dibujos de los animales estudiados en Lanzarote, y los continuó en Europa con algunos ejemplares bien conservados y datos que redescubrió en sus notas. En primer lugar, observa, analiza e ilustra el desarrollo embrionario de la especie Eurhamphaea: desde la puesta de huevos hasta la segmentación y los diferentes estadios evolutivos. Una vez contrastadas las conclusiones de Haeckel, Leuckart, Gegenbaur, Agassiz, Will, Carus, Kölliker y Claus; y cubiertas las lagunas remanentes, una de sus primeras constataciones fue que, con el conocimiento de entonces, no se podía determinar si los ctenóforos habían derivado de animales tetrarradiales u octorradiales (Fol 1869: 1-6). A continuación, aborda las especies Vexillum parallelum y Cestum Veneris Lesueur. Esta última había sido estudiada previamente por varios autores, pero las descripciones de su conducto linfático eran incompletas y divergentes, por lo que Fol ofrece una más exacta y revisada. Para la primera especie aporta su descripción: "con el nombre Vexillum designo un ctenóforo aún no descrito de la familia de los céstidos» (Fol 1869: 6), y concluye, no obstante, que sería aventurado dar el animal por nuevo y atribuirle valor de género, pues podría tratarse de «un estado joven de Cestum o de otro céstido» (Fol 1869: 8).

Considerando que nos interesa destacar coincidencias y divergencias entre nuestras cuatro tesis, tomaremos como referencia la de Fol, aún anterior al mencionado reglamento unificador de 1872. Conforme al $\$ 96$ de los Estatutos de la Facultad de Medicina de Berlín de 1838 (Koch 1839: 112-138), para presentar la tesis se requería: haber estudiado al menos cuatro años en una o en

7 Su también estudiante Nikolai N. Mikloucho Maclay y su colega Richard Greeff, entonces profesor en la Facultad de Medicina de la Universidad de Bonn. 
varias universidades; haber aprobado el examen denominado Philosophisches Tentamen, que acreditaba los conocimientos básicos en Lógica y Psicología, Zoología, Botánica, Mineralogía y, especialmente, Física y Química (\$97); pasar el examen escrito y oral de la Facultad de Medicina vigilado por el Decano (\$98) y el rigorosum pro grado Doctoris $(\$ 101)$, ante un tribunal de seis examinadores sobre las materias específicas de Medicina $(\$ 104)$. Posteriormente, el doctorando debía aportar a la Facultad, con un curriculum vitae que recogiese su credo religioso, 150 ejemplares de su disertación -de al menos dos cuadernillos de 16 páginas cada uno (zwei Druckbogen)- para su distribución en la Universidad ${ }^{8}$ y entre los oponentes. El tema debía estar vinculado a una de las ciencias naturales con relación instructiva con la Medicina. Con la disertación, que, antes de presentarse en público, había de defenderse ante el decano ( $\$ 109)$, el doctorando debía entregar una declaración escrita de que la había elaborado sin ayuda de otros $(\$ 110)$. Superados estos trámites, procedía a la defensa, que, presidida por el decano o su representante, realizaba desde la silla inferior (\$113), ante al menos tres oponentes reconocidos por la Facultad, que, si el doctorando no lograba encontrar, serían nombrados de entre sus Privatdozenten (\$115), y cuyos nombres debían figurar junto al título de la disertación.

Como cabía esperar, Fol aporta: las tesis que se proponía defender, un currículum de apenas siete líneas y los nombres de los encargados de suscitar dudas y oponer objeciones (C. A. Ruge, Dr. Med.; O. Soltmann, Dr. Med. y J. Berthoud, Cand. Theol.), de los que consideramos oportuno reseñar algunos datos. Carl Arnold Ruge y Otto Soltmann habían estudiado Medicina en Berlín con Fol y acababan de doctorarse: Soltman el 8 de mayo y Ruge el 13 de agosto de 1869 (VBU 1899: 503, 499). Ruge incluso había coincidido con Fol en Jena en 1865 (MUJ 1865: 3) y Soltmann también había cursado parte de sus estudios en Zúrich (Groger 1990: 346-347). En cuanto al tercero, debe de tratarse de Jean Berthoud, del cantón de Vaud, al lado de Ginebra, de donde era Fol, y que posteriormente fue predicador en las localidades de Savigny y Bulle, y pastor en la iglesia libre de Le Santier y de L'Isle (HBLS 1924: II, 203). Su nombre no figura en los catálogos de las universidades en las que había estudiado Fol, pero, tras haber cursado Teología y obtenido el título de Candidatus Theologiae, podría encontrarse en el periodo de formación práctica conducente al oficio pastoral en alguno de los Seminarios de Predicadores cercanos, por ejemplo, en Wittenberg (cf. Dibelius 1917).

\section{La tesis de Franz Keibel. Antropología}

Dieciocho años después de la de Fol, en 1887, se presentó en la Facultad de Medicina de la Universidad de Estrasburgo la segunda tesis, obra de Franz Keibel (1861-1929), con el título Los aborígenes de las Canarias. Una

8 El texto de Fol está redactado en 13 páginas, pero en letra menuda, con cuatro láminas dibujadas por él. 
aproximación antropológica, y supervisada por el ya renombrado anatomista, morfólogo y pionero antropólogo Gustav Schwalbe. Keibel había nacido en la localidad de Adlig Dombrowken, distrito de Graudenz, en la Prusia occidental, donde estudió el bachillerato (Peter 1929: 201). Los estudios de Medicina los realizó de 1882 a 1885 en Berlín -donde tuvo, entre otros profesores, al conocido médico patólogo Rudolf Virschow (1821-1902) (AVPSKFWU Berlin 1884: 7)- y los concluyó en Estrasburgo.

¿Por qué vino Keibel a Estrasburgo, y cuál fue realmente la motivación para su estudio craneológico de los canarios prehispánicos? Uno de los profesores cuyo apoyo agradece expresamente Keibel (1887: 57) fue Friedrich Daniel von Recklinghausen (1833-1910), que, al terminar sus estudios de Medicina en Berlín, había trabajado en el Instituto Anatómico de la capital prusiana a las órdenes de Virschow, con quien volvió como asistente en 1858, tras haber estado en París, Viena y Roma. En Estrasburgo, adonde había llegado en 1872, asumió la cátedra, la prosectura del Hospital civil y la dirección del recién fundado Instituto de Patología anatómica, en el que formó una colección de esqueletos con malformaciones (Murken 2003). Por otro lado, en Estrasburgo estaba como catedrático desde 1883 Schwalbe, que ya había dirigido dos tesis (en Jena, 1878, y Königsberg, 1883), y de quien el propio Keibel (1916: 213-214) destaca que siempre atendía las inquietudes de sus estudiantes y los animaba en sus intereses, a él particularmente en su tesis.

Schwalbe no tuvo relación directa con las islas Canarias, pero ya cuando estudió en la Facultad de Medicina de Zúrich en 1863 (MUZ) pudo haber coincidido con el geólogo Karl von Fritsch (1838-1906), que, aquel año, justo al regresar de su primer viaje a Canarias, se habilitó allí, en la Facultad de Filosofía, e impartió docencia hasta 1867 (HVUZ). Posteriormente, cuando trabajó en la Universidad de Bonn, en 1867-1868, bajo la dirección de Max Schultze (Keibel 1916: 210), a la sazón director del Instituto anatómico y del museo de aquella Facultad de Medicina, debió de conocer a Richard Greeff, también profesor allí (AVPSKRFWU Bonn, 1868: 3, 5, 6), y que había estado en Lanzarote con Haeckel poco antes ${ }^{9}$. Obviamente, en Jena, donde Schwalbe trabajó como profesor de Anatomía de 1873 a 1881 (Keibel 1916: 211), conoció a Haeckel. Por lo demás, los estudios antropológicos sustentados en análisis y mediciones craneológicos, iniciados en el ámbito germanoparlante en 1883 por el arqueólogo y antropólogo austriaco Josef Szombathy (1853-1943), a la sazón director de la colección antropológica-prehistórica del Museo de Historia Natural de Viena ${ }^{10}$, sin duda, ya eran conocidos por Schwalbe. Keibel lo cita

9 Es significativo que Keibel no cite a Greeff en el interior del texto, pero sí en la bibliografía. De aquella estancia, del 22 de noviembre de 1866 al 2 de marzo de 1867, publicó Greeff, entre otros trabajos, el extenso relato Reise nach den Canarischen Inslen, 1868 (cf. Sarmiento Pérez 2011: 38-40, 82-84).

10 Szombathy (1883: 76-77) había analizado dos cráneos guanches que el también austriaco Viktor von Schwerzenbach había encontrado en una cueva del norte de Tenerife. 
en varias ocasiones, y muy probablemente a través de él llegase a autores como Berthelot, Verneau, Quatrefages y Hamy -que adscribían los guanches a la raza Cro-Magnon. Finalmente, como antropólogo, tampoco debía de desconocer los aventurados planteamientos del médico, jurista, historiador y archivero Franz von Löher (1818-1892), que, tras visitar las Islas en 1873 por encargo del rey Luis II de Baviera, había iniciado el debate sobre la presencia germana en las Canarias al proponer su poblamiento por los vándalos en el siglo $\mathrm{V}^{11}$.

Además de analizar 36 cráneos que encontró en varios autores (Virschow, Verneau, Davis, Flower, Szombathy, Retzius, Meigs, Sprengel, Hœeven), Keibel realizó mediciones de cinco cráneos de diferentes partes del archipiélago canario que le había facilitado Schwalbe (Keibel 1887: 33): dos de ellos habían sido un regalo de Paul Langerhans (1847-1888) ${ }^{12}$, procedentes de una cueva de Tenerife, cerca de Güímar; otros dos procedían del Instituto Anatómico de Senckenberg en Fráncfort, donados por Karl von Fritsch y Johan Justus Rein ${ }^{13}$, y el quinto estaba en el Museo de Estrasburgo, al parecer llegado allí a través de Webb (Keibel 1887: 33-34, 43, 49), en cualquier caso, probablemente de la colección de Recklinghausen.

La tesis de Keibel resulta rigurosa en fondo y forma. Un detallado recorrido histórico sobre las islas Canarias desde la Antigüedad hasta su redescubrimiento a finales de la Edad Media (sociedad de los antiguos canarios, su origen, lengua, usos y costumbres, etc.), da paso a un análisis del estado de la cuestión. Sus fuentes, que presenta de forma crítica, son francesas (Berthelot, Verneau ${ }^{14}$, Faidherbe, Quatrefages, Hamy, etc.), germanas (Humboldt, Virschow, Löher, Müller, Szombathy, Pauli, Greeff, etc.), inglesas (Flower, Davis) y suecas (Retzius). Keibel lamenta no haber tenido acceso directo a las obras de Chil y Naranjo, a quien cita a través de autores franceses. Seguidamente, tras comparar el conjunto del material encontrado en los diferentes autores, y aplicando el sistema de medición de la Frankfurter Verständigung de 1882, analiza los cinco

11 Pese a la inconsistencia de su vinculación de los canarios prehispánicos con los vándalos, la aportación bibliográfica Löher es muy meritoria: dos monografías (Nach den Glücklichen Inseln. Canarische Reisetage, 1873, y Das Kanarierbuch. Geschichte und Gesittung der Germanen auf den Kanarischen Inseln, 1895) y varios artículos, $1876,1880$.

12 Langerhans había sido alumno de Haeckel en Jena y de Virschow en Berlín. En ambas universidades coincidió con Fol, y también se doctoró en Berlín en 1869 (AVPSKFWU Berlin 1869: 10, 26; VBU 1899: 497, 504). En 1878, desde Madeira, donde entonces residía, pasó algunos meses en el norte de Tenerife y estudió algunos anélidos que pescó allí (Über einige canarische Anneliden, 1881).

13 Aquellos dos cráneos, junto con otras antigüedades canarias, se los había regalado Chil y Naranjo a Fritsch y a Rein en Las Palmas en 1872 (Fritsch 1873: 14). En aquel viaje, Fritsch se reencontró con Chil, a quien había conocido, e incluso pasado algún tiempo con él en Gran Canaria entre finales de abril y principios de mayo de 1863 durante su primer viaje a las Islas (Fritsch 1867: 23, 25).

14 Sus trabajos menores, pues Cinq années de séjour aux îles Canaries no se publicó hasta 1891. 
cráneos de que dispuso, todo ello recogido en minuciosas tablas e ilustraciones (Keibel 1887: $62 \mathrm{ss,} \mathrm{s/p).} \mathrm{Entre} \mathrm{sus} \mathrm{conclusiones,} \mathrm{destaca} \mathrm{que} \mathrm{el} \mathrm{material}$ examinado no mostraba propiedades que pudieran interpretarse como signos raciales, por lo que cuestionaba que, de los resultados obtenidos hasta aquel momento, se pudiera asegurar un parentesco entre los guanches y la raza CroMagnon (1887: 51).

Frente a la de Fol, la tesis de Keibel presenta notables diferencias: en la página del título aparece el año del depósito, 1887, y la anotación «Approbierter Artzt», o sea, Médico con licencia para ejercer, pero no la fecha de presentación y defensa, ni los oponentes; en cuanto al interior, no se incluye ni el curriculum vitae ni las proposiciones (Thesen). La explicación la encontramos en los estatutos de la Facultad de Medicina de la Universidad de Estrasburgo (Baumgart 1885: 145-148), que, al respecto, solo disponían que no tendría lugar un acto público de presentación y defensa $(\$ 15)$, y que, en el momento de la inscripción para la promoción, el candidato había de entregar al decano «un trabajo científico del vasto ámbito de la Medicina» y un curriculum vitae (\$4). El decano entregaría el trabajo a un evaluador experto (Referent), quien, a su vez, junto con su dictamen escrito, lo haría circular entre los miembros de la Facultad para que lo aceptasen o lo rechazasen. Si el doctorando obtenía la aprobación, procedía a realizar el «examen de la Facultad» (\$5), oral y teórico «de todas las asignaturas importantes de la Medicina» ( $\$ 6)$, que, para quienes hubiesen estudiado en aquella universidad, consistía en un debate "ante tres miembros de la Facultad» (\$7). No obstante, Keibel (1887: 2) sí añade en el reverso de la portada una información que no aportan los otros tres doctorandos: «Impreso con la autorización de la Facultad de Medicina de la Universidad de Estrasburgo» y el nombre del experto evaluador, el «Prof. Dr. Schwalbe», como establecía el $\$ 13$. Cabe añadir que esta tesis, con 60 páginas de letra menuda y 16 de tablas e ilustraciones, es sustancialmente más extensa que la de Fol. Los estatutos de Estrasburgo no disponían nada al respecto, aunque sí coincidían con los de Berlín en que el candidato había de entregar una declaración de que la disertación la había elaborado él mismo $(\$ 4)$.

\section{La tesis de Otto Walter. Geología}

La tercera de nuestras tesis fue la presentada y defendida por Otto Walter en la Facultad de Filosofía de la Universidad de Halle-Wittenberg en 1893 [1894], consistente en una primera aproximación a las rocas de la isla de El Hierro, supervisada por Karl von Fritsch, catedrático de Geología allí desde 1876, y de Otto Lüdecke, catedrático de Mineralogía desde 1884. Walter había nacido el 26 de julio de 1857 en Halle, donde fue a la Escuela Latina. De 1877 a 1881, había estudiado Ciencias Naturales y Matemáticas en la Academia Fridericiana Hallensis. Tras aprobar, en 1883, el examen pro facultate docendi, de 1884 a 1885 realizó las prácticas como profesor de enseñanza media en el Instituto de la Fundación A. H. Francke de Halle y pasó a la Escuela Real Superior 
Guerick de Magdeburgo, donde alcanzó el nivel de catedrático (Oberlehrer). Paralelamente, siguió cultivando los estudios petrográficos por su cuenta o en el Museo Mineralógico de Halle (Walter 1894a: 125; Kössler 2008: s/p) y, a partir de 1885, cooperó en la Asociación de Ciencias Naturales de Magdeburgo (Naturwissenschaftlicher Verein zu Magdeburg): como bibliotecario, secretario de la Junta Rectora, redactor de su revista (Jahresbericht von Abhandlungen des Naturwissenschaftlichen Vereins zu Magdeburg) y, en 1892, en una comisión para la mejora de su museo, que aquel año aumentó su colección de minerales con una donación. En 1893, integró el comité organizador del 25 aniversario de la Asociación (Walter 1894b: 25, 28, 32, 38, 43).

Otro de los profesores que Walter tuvo en Halle fue el catedrático de Zoología Hermann Grenacher (1843-1923), que también había investigado en las islas Canarias del 26 de agosto al 9 de octubre de 1871, mayormente en Tenerife (Noll 1872: 21-23) ${ }^{15}$. De aquel viaje resultaron numerosas publicaciones, sobre todo en la revista de la Sociedad de Naturalistas Senckenberg (Bericht über die Senckenbergische Naturforschende Gesellschaft in Frankfurt am Main), que entonces codirigían Fritsch y Rein, y varias conferencias en colegios y otras instituciones, que, sin duda, coadyuvaban a despertar el interés científico por Canarias (Noll 1872: 26) ${ }^{16}$.

Ahora bien, quien más influyó en el ya no tan joven candidato, pues se doctoró con 36 años, fue, sin duda, Fritsch, que conocía como nadie la geología de Canarias, pues la había estudiado in situ de septiembre de 1862 a mediados de junio de 1863 y posteriormente otras cuatro semanas en 1872 en compañía de Rein (Fritsch 1864: 114; 1872: 365) ${ }^{17}$. Pero Fritsch reunía otras características que lo hacían ideal como director de tesis: disponía de material y era sumamente generoso. Como desvela el propio Walter, su maestro le había proporcionado las muestras de rocas de El Hierro para su investigación. Esto no era nuevo, pues también a Lüdecke, que, antes que profesor de Walter, había sido alumno de Fritsch, le había suministrado el material para su tesis (Der Glaukophan und die Glaukophan führenden Gesteine der Insel Syra, 1876), traído de sus viajes al

15 Grenacher viajó como primer becario de la Fundación Rüppel. Tenía entonces 29 años, era ya Privatdozent de Zoología en Göttingen y se proponía estudiar los animales inferiores (marinos y terrestres). La Fundación había encomendado al Dr. Friedrich Karl Noll (1832-1893) apoyar al joven investigador.

16 A este respecto escribía Noll (1872: 26): «[...] En el programa de Pascua de la Escuela superior ofrecí a alumnos y profesores un bosquejo de la Vegetación del Valle de la Orotava y de las Canarias; particularmente sobre la de Tenerife hablé en la Asociación del Jardín de aquí. [...] Espero que, una vez tengamos más elaborado el material que trajimos, podamos contar con otras conferencias en el próximo invierno».

17 Aquellos dos viajes dieron lugar a varias conferencias de Fritsch, así como a la publicación de artículos y algunas monografías (entre estas últimas, Reisebilder der Canarischen Inseln, 1867, Tenerife, geologisch-topographisch dargestellt. Ein Beitrag zur Kenntnis vulkanischer Gebirge, 1867, en coautoría con G. Hartung y W. Reiss, y Geologische Beschreibung der Insel Tenerife. Ein Beitrag zur Kenntnis vulkanischer Gebirge, 1868, con W. Reiss). 
Mediterráneo oriental (Lüdecke 1906: 149, 158). Además, aparte de ejercer la cátedra de Geología y dirigir el Instituto Mineralógico de Halle, Fritsch, difundía sus investigaciones en conferencias, de las que sobre Canarias podemos destacar, a modo de ejemplo, las dictadas en la Sociedad de Naturalistas Senckenberg en Fráncfort en 1870, en la Sociedad de Naturalistas de Halle el 26 de junio de 1878 y en la Asociación de Ciencias Naturales de Magdeburgo en 1885 (Fritsch 1870, 1878; Walter 1886: VI).

En su tesis, Walter analiza 39 rocas -de formación u origen volcánico-, que, como hemos dicho, le había proporcionado Fritsch: 17 pertenecientes a los basaltos feldespáticos; 11, a las basanitas; una, a las nefelinotefritas; una, a las limburguitas; una, a las andesitas hornbléndicas; seis, a las tobas volcánicas y cinco, a las palagonitas. Hasta entonces se habían estudiado las rocas de las demás islas, pero no de El Hierro, por lo que su objetivo era cubrir esta laguna. Organizado en dos partes, el trabajo abarca una relación de las características generales de los conglomerados de las rocas y sus ubicaciones en las localidades herreñas. A la aportación propiamente de Walter, precede un texto de Fritsch (p. 1-29) que describe la estructura y composición del suelo de la Isla, destinado a completar dos de sus anteriores trabajos: los mencionados Reisebilder..${ }^{18} \mathrm{y}$ el de su conferencia en Halle en 1878. En el contexto de la tesis, el aporte del «director» cumplía dos funciones: evidenciar su prestigio (cf. Rasche 2018: 294) y servir de guía en lo tocante a la geografía herreña, que Fritsch había recorrido entre el 6 de enero y el 7 de febrero de 1863.

La parte de Walter comprende 90 páginas (p. 30-125) de texto especializado, fruto de un exhaustivo análisis, en parte mediante observaciones al microscopio, y tomando como base para las denominaciones de las rocas la obra de H. Rosenbusch Mikroscopische Physiographie der massigen Gesteine, vol 2, 1877. Ahora bien, se echa en falta un estado de la cuestión o, al menos, menciones a las investigaciones precedentes (L. v. Buch, para Tenerife, Gran Canaria y La Palma; G. Hartung, para Lanzarote y Fuerteventura, o Escolar y Webb \& Berthelot para la Geología general de las Canarias). Por lo demás, solo se cita como fuente consultada la obra de C. F. Naumann Elemente der Mineralogie, 1846.

Aunque la publicase en 1894, Walter presentó su tesis en 1893, como él mismo desvela al recogerse como «Dr. O. Walter» en la relación de actividades de aquel año en su escrito sobre el 25 aniversario de la Asociación de Ciencias Naturales de Magdeburgo (Walter 1894b: 41). Esto se explicaría porque los Estatutos de la Facultad de Filosofía de Halle (Baumgart 1885: 173-176) permitían la publicación de los 200 ejemplares de la disertación hasta seis meses después de la obtención del grado de doctor $(\$ \mathrm{VII})$. Por otro lado, junto al título de la publicación no figuran: los oponentes, ni la leyenda «entregada y defendida», ni la fecha de la defensa. En este caso, la explicación radica en que, conforme al $\S$ VIII de los Estatutos, se podía renunciar a la defensa pública de la disertación y las tesis autorizadas por la Facultad, aunque si así se hiciera, ya no

18 Traducidos al español por Batista Rodríguez y Tabares Plasencia (2006). 
se podría ejercer como Privatdozent en una universidad prusiana (\$IX). Walter eligió esta opción, lo que evidencia que su objetivo no era entrar en la carrera universitaria. Cabe añadir, finalmente, que, si bien el curriculum vitae se había de entregar en latín, como así hizo Walter (1894: 125), la Facultad decidía si el examen oral se hacía en latín o en alemán, en función de las capacidades del candidato.

\section{La tesis de Walter Kampf. Economía}

La cuarta y última de las tesis que nos ocupan, sobre las fuentes de ingresos de las islas Canarias hasta finales del siglo xIx, y supervisada por el catedrático de Geografía Económica Johann Justus Rein (1835-1918), fue defendida el 4 de agosto de 1894 en la Facultad de Filosofía de la Universidad de Bonn. Walter Kampf había nacido el 19 de junio de 1866 en la localidad de Werden, cerca de Düsseldorf, en cuyo Instituto Real pasó la prueba de madurez en 1886. Luego, estudió cinco semestres en la Escuela Superior Técnica de Charlottenburg en Berlín y, tras interrumpir los estudios algo más de dos años por enfermedad, en 1891, ingresó en la universidad (Kampf 1894: 91). Cuando se doctoró tenía 28 años.

Como consta por el agradecimiento en el curriculum vitae al final de la tesis (Kampf 1894: 91), quien lo indujo a elegir el tema fue Rein, a la sazón decano de la Facultad de Filosofía, director de su Instituto Geográfico (APVRFWU Bonn 1890: 1, 7) y, entre 1880-1891, coeditor de la revista Zeitschrift für wissenschaftliche Geographie. En realidad, sus clases abarcaban prácticamente todo el ámbito de la Geografía, incluyendo historia y distribución geográfica de las principales plantas de cultivo de la región mediterránea (Hohmann 1968: 4). Además, conocía las Canarias, donde, como ya mencionamos, había estado en 1872 en compañía de Fritsch (1872: 365) ${ }^{19}$. Aunque él mismo no publicó un relato de aquel viaje, sí coeditó con Fritsch un breve informe sobre el cultivo de la cochinilla (Fritsch y Rein 1873: 49-52) ${ }^{20}$. Sus estudiantes destacaban su poder de convicción, basado en que enseñaba sobre lo visto y vivido (Ziegler 1919: 141). Impulsó otras tesis similares, por ejemplo, sobre el cultivo del arroz en Italia y del té en la India y Ceylán (Hohmann 1968: 5).

El objetivo de Kampf era analizar la evolución de las fuentes de ingresos de Canarias desde las menciones en la Antigüedad clásica. En un minucioso recorrido, a modo de estado de la cuestión, desgrana los productos cultivados

19 En Gran Canaria (del 18 de abril al 6 de mayo) recorrieron los alrededores de la capital, recogieron muestras de sedimentos portadores de petrofactos e hicieron una excursión por el centro hasta Mogán, regresando por el norte. De Tenerife (del 7 al 13 de mayo) no dicen dónde estuvieron (cf. Fritsch 1872: 365).

20 Entre las publicaciones de Rein figuran varios trabajos sobre la provincia de Huelva y Sierra Nevada, siendo su aporte más destacado Geographische und Naturwissenschaftliche Abhandlungen über Spanien (1892) (cf. Hohmann 1968: 5-7). 
o comercializados en el Archipiélago. El trabajo se estructura en tres bloques: el primero nos presenta la situación geográfica, el poblamiento, la situación político-administrativa, el redescubrimiento, la conquista y los siglos siguientes; el segundo aborda la naturaleza (geología, orografía, clima, flora -con especial atención a las plantas de cultivo-, pisos vegetales, fauna, etc.); y en el tercero se analizan uno a uno los productos (circunstancias de su introducción, avatares por los que fueron pasando, países de exportación, etc.). En definitiva: se radiografía la evolución de la economía canaria hasta finales del siglo XIX.

Recogido en 93 páginas de letra menuda, el trabajo resulta riguroso y bien documentado. Como cabía esperar, las fuentes mayoritariamente consultadas son las de lengua alemana ${ }^{21}$ de carácter general (Anderson, Benedikt) y sobre Canarias (L. v. Buch, MacGregor, Minutoli, Christ, Greeff, Löher, Noll, Ehrmann, Mannert, Wyss, incluyendo la tesis recientes de E. Wiepen y H. Lojander), pero también incluye fuentes francesas (Berthelot, Bory de SaintVincent, Dampier, Milbert, Ledru, Ogier), inglesas (Glas, Hackluyt), italianas (Benzoni) y españolas (Viera, Madoz).

En cuanto a aspectos formales, Kampf no indica los oponentes. Esto pudiera obedecer a que, si bien los Estatutos de la Facultad de Filosofía (Baumgart 1885: 158-162) dejaban claro que entre ellos habría un profesor ordinario designado por la Facultad $(\$ 44)$, no explicitaban que sus nombres debieran figurar junto al título. Sí recoge, no obstante, las cuatro tesis sobre las que había «disputado» (la primera: «Sería deseable que las Ciencias de la Tierra tuvieran más peso en la clase de Geografía en los Institutos») (Kampf 1894: 92). Cabe añadir que los Estatutos tampoco especificaban el número mínimo de páginas de la disertación, ni el de ejemplares para la Facultad, aunque sí incidían en la declaración jurada de que la disertación la había escrito el propio doctorando $(\$ 42)$, y en que nadie sin el título de doctor podía impartir docencia en la Facultad (\$49).

\section{¿Qué fue de los cuatro doctores?}

De vuelta a Ginebra, en 1870, Fol obtuvo la licencia para ejercer la Medicina, pero optó por la Zoología e instaló un laboratorio marino en Mesina. Más tarde fundó la estación zoológica de Villefranche-sur-Mer, donde transcurrieron sus años más productivos, pues, si bien en 1878 ocupó la cátedra de Embriología Comparada y Teratología en la Universidad de Ginebra, en 1886 volvió allí como director adjunto. Desde su cátedra impulsó la revista Recueil Zoologique Suisse. Publicó un manual de anatomía microscópica comparada (Lehrbuch der vergleichenden mikroskopischen Anatomie mit Einschluss der vergleichenden Histologie und Histogenie, 2 vols., 1884, 1896) y unos 140 artículos, mayormente sobre embriología y fertilización. Considerado el padre de la citología moderna, fue acogido en varias sociedades científicas, entre ellas, la Academia Leopoldina

21 Algunas incluidas en la bibliografía, pero no mencionadas en el texto (como Hermann Schacht o Victor von Meyer). 
de Naturalistas Alemanes. Del Gobierno francés recibió la Gran Cruz de la Legión de Honor. Con sólo 47 años, falleció en la costa de la Bretaña francesa en 1892 (cf. Sarmiento Pérez 2011: 35-38).

Keibel, por su parte, no volvió a abordar el tema de su tesis, pero aún en sus últimos años de vida manifestaba el deseo de visitar las Canarias. A partir de 1889 dirigió la prosectura en el Instituto Anatómico de Friburgo hasta que, en 1914, volvió a Estrasburgo como catedrático de Anatomía y director del Instituto Anatómico. En 1918, hubo de abandonar la ciudad a consecuencia de la Guerra. En sus últimos años dirigió el Instituto Anatómico-Biológico de Berlín (Peter 1929: 201-203). Su labor científica se centró fundamentalmente en la embriología, propugnando el estudio monográfico de las especies, abordando el embrión en todas las etapas y utilizando las láminas comparativas de Haeckel y His y las tablas de A. Opel. Para ello, impulsó el monumental proyecto de láminas (Normentafeln zur Entwicklungsgeschichte der Wirbeltiere, 16 vols., 1897-1938), que reproducen monográficamente series completas del desarrollo de vertebrados y fueron la base para su historia evolutiva comprada; con ellas refutó la Ley biogenética haeckeliana (Peter 1929: 212, 213).

Poco sabemos de Walter con posterioridad a la publicación de su tesis, con la que probablemente solo persiguiera progresar en la enseñanza media. Como catedrático de instituto podía ascender a director y obtener el prestigiado título de Professor, otorgado a catedráticos con cierta antigüedad y afán científico (Irmer 1902: 817-818). Sea como fuere, siguió impartiendo clases y colaborando con la Asociación de Naturalistas en Magdeburgo. Con motivo de su 25 aniversario, en 1894 escribió la correspondiente retrospectiva (Walter 1894b: 1-45) y en 1900 publicó un trabajo sobre el desarrollo de la botánica en el siglo XIX («Die Entwicklung der Botanik im 19. Jahrhundert»). Todavía en 1919 -ya con la categoría de Geheimer Studienrat, una suerte de asesor educativo- seguía en la Asociación (Mertens 1919: 178, 211, 220). A partir de aquí le perdemos la pista. Menos aún sabemos sobre Kampf. Teniendo en cuenta que había estado largamente enfermo durante sus estudios, no descartamos que falleciera al poco de doctorarse.

\section{Conclusiones}

Como suponíamos al inicio, las cuatro tesis reflejan los múltiples efectos que tuvieron las investigaciones realizadas en el Archipiélago por naturalistas, historiadores, geógrafos e incluso cónsules (MacGregor y Minutoli22), que luego se publicaban, se enseñaban en universidades o se difundían en congresos,

22 Nos referimos aquí a Francis Coleman Mac-Gregor y al Barón Julius von Minutoli. El primero, aunque de ascendencia escocesa por vía paterna, nació y se educó en Alemania. Entre 1825 y 1830 residió en Tenerife, donde ejerció como cónsul británico. Por su parte, Minutoli visitó las islas Canarias en 1853 cuando era cónsul de Prusia en España y Portugal. 
conferencias, cartas, etc. Igualmente alumbran la circulación del conocimiento sobre Canarias desde el territorio isleño hacia Alemania, y a la inversa, mediante entramados de contactos propiciados por una característica de la universidad alemana en la segunda mitad del siglo xix: la movilidad de los estudiantes y docentes-investigadores, que pasaban de una a otra con suma facilidad. Lo evidencian Fol y Keibel, pero sobre todo los impulsores de las tesis. Entre ellos resulta paradigmática la figura de Fritsch, pues participó directamente en la tesis de Walter, indirectamente en la de Keibel (mediante su contacto personal con Chil, su pertenencia a la Sociedad de Ciencias Naturales Senckenberg y su probable relación con Schwalbe), y presumiblemente en la de Kampf, pues él introdujo a Rein en las Canarias.

El material utilizado en tres de las tesis salió claramente de las islas Canarias: ejemplares de ctenóforos, cráneos aborígenes y rocas de El Hierro; pero también es probable que Rein se llevase textos utilizados luego en la tesis de Kampf, acaso por recomendación o cesión de Chil, a quien conoció en 1872 en Las Palmas, o de Berthelot, con quien Fritsch contactó en Tenerife e intercambió correspondencia (cf. Le Brun 2016: 325). La salida de material hacia Alemania no obedeció únicamente al expolio incontrolado de los naturalistas y eruditos que visitaban las Islas, sino también a lazos de amistad y cooperación entre ellos e instituciones y eruditos canarios, que solidificaban relaciones de ida y vuelta, mediante: cartas, intercambio de publicaciones, citas y menciones. No es este lugar para profundizar en ello, pero sí podemos ejemplificarlo: a través de Berthelot y de Chil, hizo llegar Fritsch, en septiembre de 1869, un ejemplar de su obra Geologische Beschreibung der Insel Tenerife a la Sociedad de Amigos del País de Las Palmas (AMC/GCh-0104). El propio Fritsch solicitó a Chil por carta, desde Tenerife en 1872, el envío de un ejemplar de Spirula que algún pescador canario capturase en la costa africana, ofreciéndole a cambio introducirlo como miembro en sociedades científicas alemanas (AMC/GCh-0127). Por su parte, Chil cita con frecuencia a su "particular amigo el barón Dr. K. Von Fritsch» (1876: XI-XII, 59, 61, 338-339, 343, 368, 385; 1880: 110, 200, 201, 285, 286). Y también lo hace Berthelot en sus Antiquités Canariennes (1879: 138-140).

No menos interesantes nos parecen otras concurrencias que traslucen las cuatro tesis. Por un lado, en el periodo que nos ocupa confluyeron en la Universidad de Bonn, al menos, tres profesores que investigaron en Canarias: además de Rein y Greeff, también lo había hecho, en 1890, el ornitólogo Alexander König (1858-1949), que trabajaba en su Facultad de Filosofía cuando Kampf realizó su tesis (APVRFWU Bonn, 1894: 8); y en Halle coincidieron Fritsch y Grenacher. Por otro lado, en las facultades de Medicina de Würzburg o Berlín tuvieron como profesor a Rudolf Virschow: Haeckel, Greeff, Recklinghausen, Langerhans, Schwalbe y Keibel. Finalmente, en esta serie de confluencias cabe recordar que de Haeckel -quien, sin duda, propició la primera de las tesis-, fue compañero de estudios Greeff, alumno Fol y compañero de trabajo Schwalbe; y Keibel refutó la Ley biogenética, para la que el sabio de Jena había encontrado fundamentos en Lanzarote (cf. Sarmiento Pérez 2011: 59, 62, 91). 
En cuanto a las tesis propiamente dichas, son de destacar algunas diferencias: las de Fol y Otto resultaron de trabajo de campo en las Islas y análisis de laboratorio en Alemania; por su parte, las de Keibel y Kampf revelan el transvase de informaciones recogidas en el archipiélago canario y publicadas en Alemania, al tiempo que la consolidación del interés de la ciencia alemana por Canarias y de los conocimientos al respecto entre docentes-investigadores e instituciones científicas. De estas dos últimas cabe añadir, finalmente, que, en consonancia con la progresiva mejora de las tesis alemanas -que alcanzó un momento álgido en la década de 1970 (Rasche 2018: 296)-, muestran ya un mayor rigor académico que las dos primeras: en la estructura, amplitud y exhaustividad de los temas tratados, así como en el tipo, la calidad y el número de fuentes consultadas.

\section{Bibliografía}

Baumgart M., 1885, Grundsätze und Bedingungen der Ertheilung der Doctorwürde bei allen Facultäten der Universitäten des deutschen Reiches, Berlin, R.V. Decker.

Berthelot S., 1879, Antiquités Canariennes, Paris, Ed. Plon.

Chesa Ponce N., 2015, La medicina en Canarias en el siglo XIX: médicos canarios formados en Francia, Madrid, Mercurio.

Chil y Naranjo G., 1876, Estudios Históricos, Climatológicos y Patológicos de las Islas Canarias, Primera Parte, Historia, Tomo Primero, Las Palmas de Gran Canaria, Isidro Miranda.

Chil y Naranjo G., 1880, Estudios Históricos, Climatológicos y Patológicos de las Islas Canarias, Primera Parte, Historia, Tomo Segundo, Las Palmas de Gran Canaria, Isidro Miranda.

Dibelius O. v., 1917, Das Königliche Predigerseminar zu Wittenberg 1817-1917, Berlin, Runge.

Fol H., 1869, Ein Beitrag zur Anatomie und Entwickelungsgeschichte einiger Rippenquallen, Inaugural-Dissertation zur Erlangung der Doctorwürde in der Medicin und Chirurgie der medicinischen Facultät der Friedrich-WilhelmsUniversität zu Berlin, Berlin, Büxenstein.

Fritsch K. v., 1864, «Zur Geologie der Canaren», Zeitschrift der deutschen geologischen Gesellschaft, 16, p. 114-120.

Fritsch K. v., 1867, Reisebilder der Canarischen Inseln, Gotha, Justus Perthes.

Fritsch K. v., 1870, «Über die ostatlantische Inselgruppen», Bericht über die Senckenbergische Naturforschende Gesellschaft in Frankfurt am Main, Juni 1869-Juni 1870, p. 72-113.

Fritsch K. v., 1872, «Reise der Frankfurter Naturforscher Dr. K. v. Fritsch und Dr. J. J. Rein nach den Canarischen Inseln und dem Marokkanischen Atlas 1872. Vorläufiger Bericht», Petermann's Geogr. Mitteilungen 18, p. 365-367. 
Fritsch K. V., 1873, «Bericht über die Senckenbergische naturforschende Gesellschaft», Bericht über die Senckenbergische naturforschende Gesellschaft in Frankfurt am Main, Juni 1872-Juni 1873, p. 3-20.

Fritsch K. v., 1878, «Hierro», Leopoldina, XIV 1-2, p. 61-64.

Fritsch K. v., 2006, Las Islas Canarias. Cuadros de viaje, Traducción, Estudio Introductorio y Notas de J.J. Batista Rodríguez y E. Tabares Plasencia, Tenerife, Centro de la Cultura Popular Canaria.

Fritsch K. v., Rein J. J., 1873, «Gegenwärtiger Stand der Cochenille-Zucht auf den Canarischen Inseln», Jahresbericht des Frankfurter Vereins für Geographie und Statistik, 36. Jg., 1871-1872, Frankfurt a. M., Mahlau \& Waldschmidt, p. 49-52. Groger H., 1990, «Otto Stoltmann», in Ashwal S. (ed.), The founders of child neurology, San Francisco, Norman Publishing, p. 346-353, <https://bit. ly/2X3Bm6Q >, consultado el 27/03/20.

Hohmann J., 1968, «Johannes Justus Rein», Erdkunde, 22-1, p. 1-7.

Horn E., 1893, Die Disputationen und Promotionen an den Deutschen Universitäten vornehmlich seit dem 16. Jahrhundert, Leipzig, Harrassowitz.

Irmer B. (Hrsg.), 1902, Das höhere Schulwesen in Preussen, 4. Bd. (1874-1901), Berlin, Viegant \& Grieben.

Kampf W., 1894, Die Erwerbsquellen auf den Kanarischen Inseln und ihre Wandlungen, Inaugural-Dissertation zur Erlangung der Doctorwürde bei der hohen philosophischen Fakultät der Rheinischen Friedrich-WilhelmsUniversität zu Bonn, Bonn, Heydorn.

Keibel F., 1887, Die Urbewohner der Canaren. Ein anthropologischer Versuch, Inaugural-Dissertation der Medizinischen Fakultät der Kaiser-WilhelmsUniversität Strassburg, Strassburg, Heitz.

Keibel F., 1916, «Gustav Albert Schwalbe», Anatomischer Anzeiger, 49, p. 210-221.

Koch J.F.W., 1839, Die Preussischen Universitäten. Eine Sammlung der Verordnungen, welche die Verfassung und Verwaltung dieser Anstalten betreffen, vol. 1, Berlin, Possen und Bromberg, Ernst Siegfried Mittler.

Kössler F., 2008, Personenlexikon von Lehrern des 19. Jahrhunderts. Berufsbiographien aus Schul-Jahresberichten und Schulprogrammen 18251918 mit Veröffentlichungsverzeichnissen, Band: Waag-Wytzes, <https://bit. ly/2yPgyX7>, consultado 10/04/20.

Le Brun N., 2016, Un francés entre guanches, Sabino Berthelot y las Islas Canarias, Santa Cruz de Tenerife, Le Canarien Ediciones.

Lüdecke O., 1906, «Karl von Fritsch. Nekrolog», Zeitschrift für Naturwissenschaften, 78 (06), p. 145-165.

Marti H., 2018, «Disputation und Dissertation in der Frühen Neuzeit und im 19. Jahrhundert - Gegenstand der Wissenschaftssprachgeschichte?», in Prinz M., Schiewe J. (eds.), Vernakuläre Wissenschaftskommunikation: Beiträge zur Entstehung und Frühgeschichte der modernen deutschen Wissenschaftssprachen, Berlin-Boston, De Gruyter, p. 271-292. 
Mertens A., 1919, «Bericht über die Festsitzung des Naturwissenschaftlichen Vereins zu Magdeburg zur Feier seines 50jährigen Bestehens am 14. September 1919», in Festschrift zur Feier des fünfzigjährigen Bestehens des Naturwissenschaftlichen Vereins zu Magdeburg. Abhandlungen und Berichte aus dem Museum für Natur- und Heimatkunde und dem Naturwissenschaftlichen Verein, vol. 3, Heft III, p. 165-222.

Murken A. H., 2003, «Recklinghausen, Friedrich von», Neue Deutsche Biographie 21, p. 236-237, <https://bit.ly/2T8Ni4C>, consultado el 17/02/20.

Noll F. C., 1872, «Mittheilung von Dr. F. C. Noll, seine Betheiligung an der ersten Reise für die Rüppell-Stiftung betr., beim Jahresfeste, den 26. Mai 1872», Bericht über die Senckenbergische Naturforschende Gesellschaft, p. 21-26.

Paletschek S., 2002, «Die Erfindung der Humboldtschen Universität. Die Konstruktion der deutschen Universitätsidee in der ersten Hälfte des 20. Jahrhunderts», Historische Anthropologie, 10, p. 183- 205.

Peter K., 1929, «Franz Keibel. Ein Nachruf», Anatomischer Anzeiger, 68, p. 201-220. Rasche U., 2018, "Wissenschaft, Praxis und Prestige. Zur Geschichte von Dissertation und Promotion», Forschung \& Lehre, 4 (18), p. 294-296.

Sarmiento Pérez M., 2011, La expedición cientifica de Ernst Haeckel a Lanzarote (1866-67). Las Canarias en la Teoría de la Evolución, Málaga, Libros ENCASA.

Szombathy J., 1883, «Prähistorische Gegenstände von den Canarischen Inseln», Mitteilungen der Anthropologischen Gesellschaft in Wien, 13, p. 75-77.

Walter O. (Red.), 1886, "Vorträge», Jahresbericht von Abhandlungen des Naturwissenschaftlichen Vereins zu Magdeburg, Magdeburg, Faber'sche Buchdruckerei, p. V-VI.

Walter O., 1894a, Petrographische Studien an Gesteinen der Insel Hierro, InauguralDissertation zur Erlangung der Doctorwürde bei der philosophischen Fakultät der Vereinigten Friedrichs-Universität Halle-Wittenberg, Halle.

Walter O., 1894b, «Geschichte des Naturwissenschaftlichen Vereins zu Magdeburg 1869-1894», in Otto Walter (Red.), Festschrift zur Feier des 25jährigen Stiftungstages des Naturwissenschaftlichen Vereins zu Magdeburg, Magdeburg, Faber'sche Buchdruckerei, p. 1-45.

Ziegler J., 1919, «Johannes Justus Rein (1835-1918)», Bericht der Senckenbergischen Naturforschenden Gesellschaft in Frankfurt a. Main, 49, p. 139-142.

Fuentes secundarias

AMC/GCh (Archivo de El Museo Canario/ Gregorio Chil).

AMC/GCh-0104. Carta de Sabino Berthelot a Gregorio Chil y Naranjo desde Sta. Cruz de Tenerife el 16 septiembre de 1869.

AMC/GCh-0127. Carta de Karl von Fritsch a Gregorio Chil y Naranjo desde Sta. Cruz de Tenerife el 8 de mayo de 1872. 
APVRFWU Bonn (Amtliches Personal-Verzeichnis der Rheinischen FriedrichWilhelms-Universität zu Bonn) für das Sommerhalbjahr 1890 u. 1894, Bonn, Georgi, <https://bit.ly/3cMugII>, consultado el 17/04/20.

AVPSKFWU Berlin (Amtliches Verzeichnis des Personals und der Studierenden der Königlichen Friedrich-Wilhelms-Universität $\mathrm{zu}$ Berlin) auf das Sommerhalbjahr 1869 u. 1884, Berlin, Buchdruckerei von Gustav Schade, $<$ https://bit.ly/2zJCJy4>, consultado el 28/04/20.

AVPSKRFWU Bonn (Amtliches Verzeichnis des Personals und der Studirenden der Königlichen Rheinischen Friedrich-Wilhelms-Universität zu Bonn) für das Sommerhalbjahr 1868, Bonn, Georgi, <https://bit.ly/2zIaPCe>, consultado el 24/04/20.

HBLS (Historisch-biographisches Lexikon der Schweiz), 2. Bd., 1924, Neuenburg, Administration des historisch-biographischen Lexikons der Schweiz.

HVUZ (Historische Vorlesungsverzeichnisse der Universität Zürich), SommerSemester 1868, <https://www.histvv.uzh.ch/vv/1868s.html>, consultado el $09 / 03 / 20$.

MUJ (Matrikel der Universität Jena) 1865 - 2. Semester, <https://bit.ly/2WTQPVm>, consultado el 23/03/20.

MUZ (Matrikeledition der Universität Zürich) 1833-1924, <https://bit.ly/2ABJlil>, consultado el 21/04/20.

VBU (Verzeichnis der Berliner Universitätsschriften) 1810-1885, nebst einem Anhang enthaltend die ausserordentlichen und Ehren-Promotionen, 1899, Berlin, Commissions-Verlag von W. Weber. 